\title{
Quality and European Programme Design in Higher Education
}

\section{Julia González and Robert Wagenaar}

The relationship between internationalisation and quality in Higher Education has been taken for granted, debated, and closely linked together at the conceptual level. It is at the conceptual level where the connection between internationalisation and quality seems to be better recognized.

The search for this link has already a significant history of studies and projects. This search is well documented in "Trends in quality assurance for European Higher Education" (Campbell and van der Wende, 2000). Furthermore, a number of conclusions can be considered of great interest as possible means to advance towards the development of the full potential of the link between quality and internationalisation.

In this respect, the report further found that it was difficult to evaluate the contribution of internationalisation to the quality of education, that the quality of internationalisation itself was not monitored or assessed systematically and that groups and actors involved in each of the processes were often disconnected. "The fact that the quality of internationalisation activities is not assessed systematically was, in the past, due to weak meassures at programme and institutional level" (Campbell and van der Wende, 2000: 6). The fact that internationalisation is not fully covered by quality assurance procedures dealing primerily with the core functions of education and research was already recognized (van Damme, 1999) and is a daily experience of any conciencious actor in the internationalisation arena. Although conceptually linked, they may be seen as two if not conflicting at least unrelated targets with different territories, in the case of the quality bodies, normally their territory relates to the national, while what goes beyond the national has become very real and growing in strength and significance, and remains uncovered in a systematic manner. This has created important difficulties for the relevant development of cooperation and in particular for crucial actions such as international joint degrees.

Following the conclusion that the quality of internationalisation activities lacks systematic quality assessment due, in part, to weak measures at programme and institutional level, two paths are clearly marked for development: institutional and programme levels.

Institutional level is an obvious and clear route to follow and significant developments have been done along these lines. From the Salamanca Convention, this approach has marked the work of the EUA and the search for quality culture at the intitutional level is one of the lines of work of this Association. In the Bologna Seminar on quality assurance in Higher Education, André Sursock focused the attention on the capacity of quality assurance mechanisms to be used by universities themselves to steer internally the institution. This is further developped at the Graz Convention where it is considered essential that universities develop an internal quality culture to monitor all their activities and services in a way that is congruent with core academic values.

It is evident that the dimension of quality at the institutional level could encompass the quality of programmes. But institutions carry a great variety within themselves, a variety which is desired and fostered to better cope with the different targets and social needs they are called to serve. Institutions do not produce programmes in series, each has its own specificity and purpose.

Besides, while institutions remain in full repsonsability of all their programmes and therefore in need to ensure their quality, the specificity of programmes which are built in cooperation, in relation with others, has, by nature, an element of transnationality which goes beyond and requires further frameworks to be understood and analysed.

The approach which will be proposed here is being developped from the project Tuning structures in Higher Education. It is in the process of being developped because the second phase, where specifically quality is presented, has just been started. Here are the initial reflections on the developping paths. Contrast with a task force made of internal and external 
experts and the reflection is still to come. So is the work and debate of over 140 experts from 25 countries. This will provide international insight, validation and realism to make it a real tested proposal to gain experience in the road to learn and advance.

The implication here is that each programme is a unit with its own ethos. Therefore quality indicators should be inbuilt as a normal and substantial part and from "within" in the sense that these indicators are not standardised norms but criteria which will respond to the uniqueness and the consistency of the original thought. They have the capacity to serve as the marks of identity of the programme where, in dynamic growth, these elements could serve as key features to return and refer.

In this respect, Tuning is searching for the quality dimension which is in agreement with the inner elements that are essential to the project. One of the definitions of quality relates to transparency; transparency of purpose, of process and of outcomes (Peter Williams, 2002). Another perspectives bring about reference of quality in terms of fitness-adequancy to purpose, to process to achieve the objectives or to be in a particular context. In the Salamanca Convention, quality was considered as a fundamental element, the basic condition for trust and relevance among other characteristics. Pelevance in terms of employability and citizenship; relevance also in terms of preparation of graduates for crucial issues to participate, work and live in a permanently changing society. This needs to be one of the points of analysis in the creation of programmes and units through constant reflection and innovation.

In the attempt to create the initial framework for developing a path for agreeing in quality meassures in European programme design in the Tuning project, transparency, fitnessadequancy and relevance will be here related to purpose, process, outcomes and approach and finally context.

\section{Quality and Purpose}

Tuning seeks to open and approach educational structures in Europe, by opening a debate aimed to identify and exchange information and to mprove European collaboration in the development of quality, effectiveness and transparency. Tuning does not seek to develop any sort of unified, prescriptive, or definitive European curricula, nor does it want to create any rigid set of subject specifications, to restrict or direct educational content and/or to end the rich diversity of European higher education. Furthermore, it does not want to restrict the independence of academics and subject specialists or to damage local and national autonomy.

When developing the project the following main aims and objectives were identified:

- To bring about a high level of Europe-wide convergence in Higher Education in the five, later seven, main subject areas (Business, Chemistry, Education Sciences, Geology, History, Mathematics and Physics) by defining commonly accepted professional and learning outcomes.

- To develop professional profiles and desired learning outcomes and competences in terms of generic competences and subject-related competences including skills, knowledge and content in the seven subject areas.

- To facilitate transparency in the educational structures and to further innovation through communication of experience and identification of good practice.

- To create European networks able to present examples of good practice, encouraging innovation and quality in the joint reflection and exchange, also for other disciplines.

- To develop and exchange information in relation to the development of curricula in the selected areas, and develop a model curriculum structure expressed in reference points for each area, enhancing the recognition and European integration of diplomas. 
- To build bridges between this network of universities and other appropriate qualified bodies in order to produce convergence in the selected subject areas.

- To elaborate a methodology for analysing common elements and areas of specificity and diversity, and for finding ways to tune them.

- To associate with other subject areas where a similar process can be incorporated through synergy.

- To act in a co-ordinated manner with all the actors involved in the process of tuning educational structures, in particular the Bologna follow-up group, Ministries of Education, Conferences of Rectors (including the EUA), other associations (as EURASHE), Quality Assurance Organisations and Accreditation Bodies, as well as universities.

Within these general objectives of the Tuning project, the European programmes are the building focal unit, and, consequently, quality in purpose in programme design would be marked by:

- Capacity to converge by defining commonly accepted learning outcomes in terms of generic and subject related competences, including skills, knowledge and content.

- Capacity to define professional profiles with relevance to employment and citizenship.

- Capacity to facilitate transparency and further innovation through communication of experiences, joint reflection and identification of good practices.

- Capacity to develop curricula not in isolation but in relation to European reference points, with European universities and with other appropiate qualified bodies.

- Capacity to follow the Tuning methodology for the analysis of what is common, diverse and dynamic in a thematic area.

- They would be programmes which include consultation with all relevant actors.

- They would be born of social needs and programmes relevant for employment as well as for citizens to take an active role in society.

\section{Quality and Process}

One of the contributions of the Tuning Project may be considered as the joint creation of a model, which is a model of process built, with a common language as much as the steps have been worked together and named after the joint experiences had taken place. Transparency relates to commonly develop language which corresponds to a jointly elaborated experience.

The purpose of the Tuning Model (see figure 1) relates to the steps in the developement of European comparable degrees. The adequacy, consistency, and relevance of the process could be further debated and analysed but a profession is clearly shown.

European Programmes leading to comparable degrees could be seen as consisting of the following steps:

1- Identification of social needs.

In the perspective of Tuning, the process in the development of a programme starts with the perception of a need. The perception of a potential or the identification and location of sources (academic, organisational, financial or strategic) can take place either parallel in time or as a consequence of the need felt.

But the social need, understood in a broader sense of a shortage of professionals, a new type of profile, etc, needs not only be perceived but tested and validated in terms of consultation with professionals and professional bodies, employers and other stakeholders, as well as in relation to the academic community. It is in this context that Tuning incorporates the consultation with European common points of reference; because comparability and compatibility are at their best when they are in built from the start.

The belief in the importance of consultation was so strong in Tuning that the project has built in a process of consultation as a proof of the importance given to this element in the process. 
THE TUNING MODEL

FOR EUROPEAN COMPARABLE DEGREES

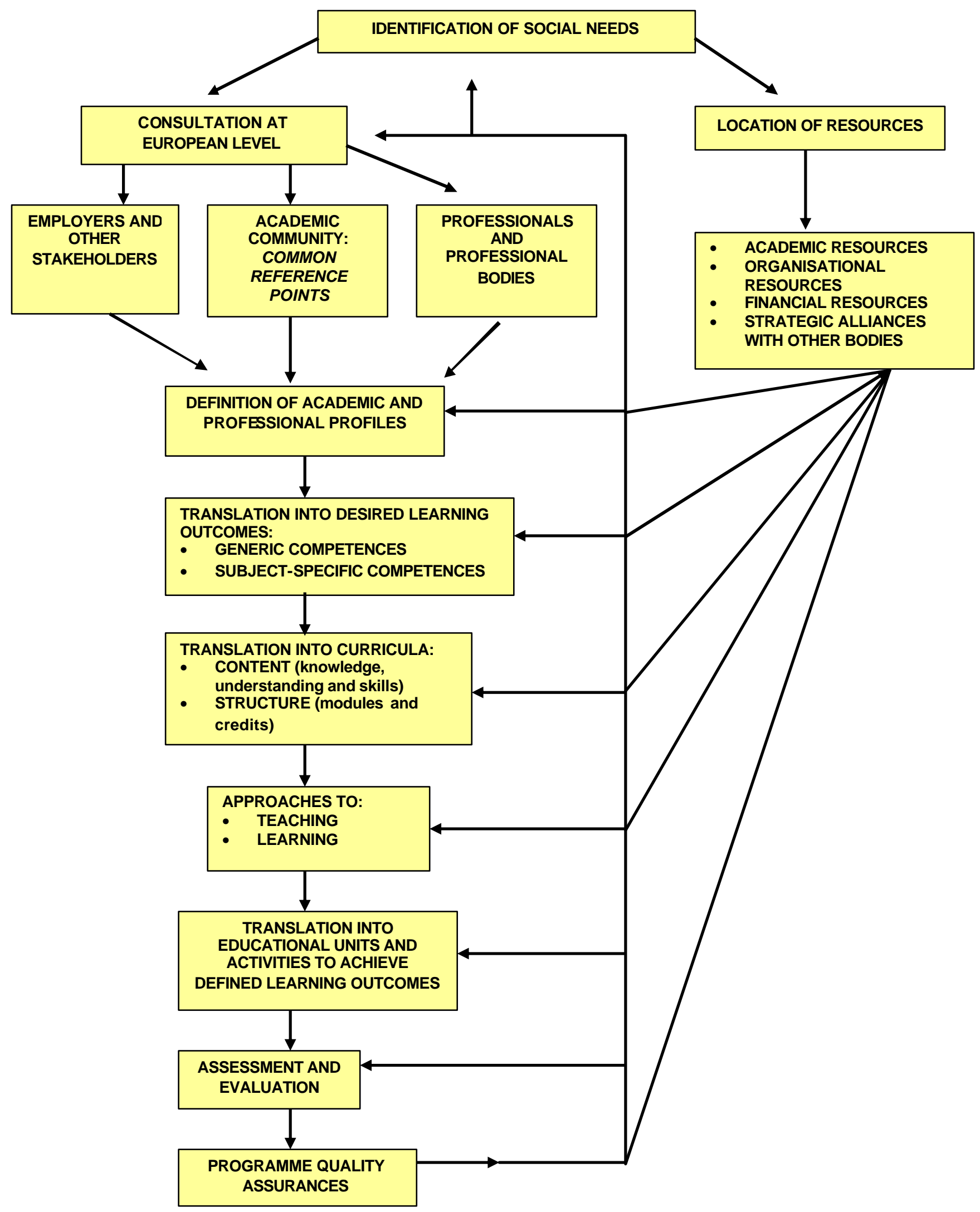


- Consultation responded to the wish to initiate the joint discussion on the field of competences and skills at the European level, based on consultation with groups from outside academia (graduates and employers) as well as from a broader base in relation to academics (beyond Tuning representatives from each of the subject areas involved).

- It was also an attempt to gather updated information for reflection on possible trends and the degree of variety and change all over Europe.

- It also responded to the desire to start from the experience and the reality in order to reach levels of diversity or commonality between the different countries, starting the debate from specific questions with concrete language.

- It wanted to prove the importance of bcusing the reflection and debate at three different levels: the institutional level (the basis and the first one to take place), the subject area level (a reference point for the HE institutions) and the aggregate level (a second reference point in relation to the situation at European level).

2- Definition of Academic and Professional profiles and the translation of these profiles into Generic and Subject - Specific competences.

In a competence-based-student-centred approach to learning, the definition of the profile of the professional clearly relates to the focus on the outcomes. The quality in terms of clarity and transparency will have a significant impact on the adequacy and consistency of the rest of the programme. Quality and transparency in the identification and definition of generic (instrumental, relational, systemic) competencies and the more specific subject related competencies which will compose the profile of a particular degree will concentrate the potential and the resources in the best use to achieve the end.

3- Translation into curricula in terms of content understood as knowledge, understanding and skills as well as structure in terms of modules and credits.

Traditionally, programme design started at the writing of curricula; and even more specifically with content, the content of the programme as designed by the teacher was the core element in a programme, which could be as such considered teacher-centred. In an outcome-based or competence-based, student-centred programme, there is a change design of curricula, the content, the modules, defined elements within a programme of study, and the credits which specify the learning which will be assessed and which normally measures the national study hours expected to be required by a student to achieve the outcomes. All these elements follow and are guided by the professional profile which the learner would be expected to attain.

4- Translation into educational modes, contexts and activities to achieve defined learning outcomes, these could adequate a significant variety. If a set of competences can have a variety both in terms of content and structure as a means to reach them the approaches to teaching and learning could reach another level of diversity. Many different paths could lead to the development of a concrete profile. These varieties should be kept and fostered.

5- Assessment and programme quality assurance.

Focusing on the assessment in relation to learning outcomes, two elements are often recognised (David Gosling and Jenny Moon, 2001): The method of assessment and the assessment criteria in clear reference to the learning outcomes.

Finally, these are some of the in-built mechanism for quality assurance within the programme with the development of indicators of consistency of process, relevance and transparency of every step and of the whole process in relation to both the contextual needs an defined profiles. 


\section{Quality and the outcomes and approaches}

Perhaps the most important outcome which emerged from Tuning is that the creation of a European Area of Higher Education in relation to Educational Structures is possible. Tuning shows that convergence fully respecting diversity can be achieved and can lead to further reflection and quality in Higher Education.

One of the key objectives of the Tuning project was to contribute to the development of easily readable and comparable degrees as well as to the understanding, "from inside", and in a European joint manner, of the nature of each of the two cycles described by the Bologna process.

In searching for perspectives which would facilitate mobility of professionals and degree holders in Europe, the project tried to reach Europe-wide consensus in the understanding of degrees from the point of view of what these holders would be able to perform. In this respect, two choices marked the project from the start: the choice to reach common points of reference and the choice to focus on learning outcomes: competences and skills (always based on knowledge).

The choice to use common points of reference and not degree definitions shows a clear positioning along three complementary lines: if professionals are to move and be employed in different countries of the European Union, their education needs to have certain levels of consensus in relation to some commonly agreed landmarks recognised within each of the subject-specific areas.

Besides, the use of reference points makes provision for diversity, freedom and autonomy. These conditions can be maintained by selecting and combining crucial elements in different ways, by taking complementary or alternative options, by following different paths etc. Diversity, freedom and autonomy mark European identity and could never be left out in a truly European project.

The provision of reference points also accommodates for dynamism. These agreements are not written in stone but are constantly developing in an ever-changing society.

Another significant feature of Tuning is the choice to look at degrees in terms of learning outcomes and particularly in relation to competences. The Tuning project deals with two types of competences: generic competences (instrumental, interpersonal and systemic) and subjectspecific competences (including skills and knowledge. First and second cycles have been described in terms of agreed and dynamic reference points: learning outcomes and competences to be developed/achieved. The beautiful thing of comparable competences and learning outcomes is that they allow flexibility and autonomy in the construction of curricula. At the same time, they are the basis for formulating commonly understood level indicators.

In this respect, while the subject area related competences are crucial for any degree and refer to the specific attributes of a field of study, the generic competences identify shared attributes which could be general to any degree, such as the capacity to learn, decision making capacity, project design and management skills, etc. which are common to all or most of the degrees. In a changing society where demands tend to be in constant reformulation, these generic competences and skills become of great importance. Furthermore, most of them can be developed, nourished or destroyed by appropriate or inappropriate learning/teaching approaches or materials.

The choice for competences as dynamic reference points in the Tuning project makes a contribution as regards to further transparency in academic and professional profiles in degrees and study programmes and a growing emphasis on outcomes

In the reflection on academic and professional profiles, competences emerge as a guiding principle for the selection of the kind of knowledge that may be appropriate to specific purposes. It has an in-built capacity to choose what is appropriate from a wealth of possibilities. 
The emphasis on students getting a specific competence or set of competences may also affect the transparency in the definition of the objectives set up for a specific educational programme. It does so by adding indicators that can be measured accountably, while making these objectives more dynamic and responsive to the needs of society and employment. This shift normally leads to a change in the approach to educational activities, teaching materials and a great variety of educational situations, since it fosters the systematic involvement of the learner, individually and in groups, in the preparation of relevant contributions, presentations, organised feedback, etc.

Besides, the shift in emphasis from input to output is reflected in the evaluation of student performance, moving from knowledge as the dominant (even the single) reference to (include) assessment centred on competences, capacities and processes. This shift is reflected in the assessment of work and activities related to student development towards pre-defined academic and professional profiles. This shift is also shown in the variety of approaches to assessment (portfolio, tutorial work, course work...) being used, as well as in situational learning. The use of competences and skills (together with knowledge) and the emphasis on outputs adds another important dimension that can balance the weight given to the length of study programmes.

The definition of academic and professional profiles in degrees is intimately linked with the identification of competences and skills and decisions on how students should attain them within a degree programme. To reach this aim, the work of isolated academics is not sufficient. The issue needs to be approached in a transversal way through the curricula of a particular degree programme.

Transparency and quality in academic and professional profiles are major assets in relation to both employability and citizenship, and the enhancement of quality and consistency as a joint effort should be a priority for the European Institutions. The definition of academic and professional profiles and the development of the fields of required competences, add quality in terms of focus and transparency.

\section{Transparency, adequancy and relevance in an international and European context}

Higher Education is characterised by rapid change. A series of general factors such as globalisation, the impact of information and communication technologies, the management of knowledge and the need to foster and managed diversity, among others, make for a significantly different environment for education. Any reflections on the future developments of education must be placed in this context. The challenges of this change and the nature of these forces, as well as the speed with which they take place, have been widely documented in the literature and referred to by European Fora, International Organisations, and papers of the European Commission.

A change is taking place in the teaching/learning paradigm, where approaches centred on the learner are increasingly important.

The "society of knowledge" is also a "society of learning". This idea is intimately linked with the understanding of all education in a wider context: the continuum of lifelong learning, where the individual needs to be able to handle knowledge, to update it, to select what is appropriate for a particular context, to learn permanently, to understand what is learned in such a way that it can be adapted to new and rapidly changing situations.

The need to recognize and value learning could also be seen as having an impact on qualifications and on the building of educational programmes leading to degree qualifications. In this context, the consideration of competences side by side with the consideration of knowledge offers a number of advantages which are in harmony with the demands emerging from the new paradigm.

\footnotetext{
${ }^{1}$ European Commission DG XXII, 1995, 1997. Comission Staff working paper, 2000. Council of the European Union, 2001. Confederation of European Union Rectors' Conferences, 2001. Thomas, E., 2000. Haug, G \& Touch, C., 2001. Mallea, J., 1998. Van Damme Dirk, 2001.
} 
In the reflection on academic and professional profiles, competences emerge as an important element which can guide the selection of knowledge which is appropriate to particular ends. It presents an integrative capacity to choose what is appropriate from a wealth of possibilities.

Trends are complex, often discontinuous processes whose effects on actors vary. However, the trend towards a "learning society" has been widely accepted and consolidated for some time. This involves a move from teaching-centred to learning-centred education. Reflecting on the different aspects which characterise this trend, the relevance of focusing on competences becomes apparent. The previous paradigm involved an emphasis on the acquisition and transmission of knowledge. Elements in the changing of this paradigm include ${ }^{2}$ : education centred on the student, the changing role of the teacher, further definition of objectives, change in the approach to educational activities, shift in emphasis from input to output, and a change in the organization of learning. Each of these elements will be discussed in turn.

The interest in the development of competences in educational programmes is in accordance with an approach to education as primarily centred on the student and his/her capacity to learn, demanding more protagonism and higher quotas of involvement since it is the student who ought to develop the capacity to handle original information and access and evaluate information in a more varied form (library, teacher, internet, etc.)

This relates implicitly with the changing role of the teacher, from being the structurer of knowledge, the key player in the teaching and articulation of key concepts, as well as the supervisor and director of work of the student, whose knowledge he/she assesses. A studentcentred vision gives the teacher more of an accompanying role, so that the learner attains certain competences. While the role continues to be critical, it shifts more and more towards containing higher levels of advice, counselling and motivation in relation to the importance and place of areas of knowledge, understanding and capacity to apply that knowledge, in relation to the profile which needs to be attained, personal interests, gaps and capacities, critical selection of materials and sources, organization of learning situations, etc.

The emphasis on students getting a particular competence or set of competences may also affect the transparency in the definition of objectives set up for a particular educational programme, adding indicators with higher possibilities for being measured accountably, while making these objectives more dynamic in taking into consideration the needs of society and employment.

This shift normally relates to a change in the approach to educational activities, teaching material and a great variety of educational situations, since it fosters the systematic involvement of the learner with individual and group preparation of relevant issues, presentations, organized feedback, etc.

Besides, the shift in emphasis from input to output ${ }^{3}$ is reflected in student evaluation, moving from knowledge as the dominant (even the single) reference to include assessment centred on competences, capacities and processes closely related to work and activities as related to student development and in relation to academic and professional profiles already defined, also showing a greater wealth of assessment strategies (portfolio, tutorial work, course work...) as well as taking into consideration situational learning.

Finally, different ways of participating in education (full time, part time...) changing contexts and diversity also affect the pace or rhythm at which individuals and groups can take part in the educational process. This also has an impact not only on the form and structure of programme delivery but in the whole approach to the organization of learning, including more focused programmes, more short courses, more flexible course structures, and more flexible delivery of teaching, with the provision of more guidance and support. ${ }^{4}$

\footnotetext{
${ }^{2}$ Villa Aurelio, 2001

| ${ }^{3}$ Report of the Engineering Synergy Group, 2002._Tuning Educational Structures in Europe

${ }^{4}$ Thomas Edward, 2000.
} 
The following requires special mention: In the context of the Bologna Declaration and the Prague Communiqué, the creation of the European Education Area responds to a number of opportunities and needs which are very relevant for European society in general and for Higher Education in particular. Among the more pressing needs, there are deepening the cooperation among the European Higher Education Institutions, with all the potential this holds; increasing the competitive edge of Europe in terms of Higher Education, particularly with a clearer and consistent picture of educational systems and the need to create the setting for the free mobility of professionals at the European level.

One of the expressions in the Declaration which refers to these needs is the development of easily readable and compatible degrees. The Tuning project considers that degrees would be comparable and compatible if what the degree holders are able to perform is comparable and if their academic and professional profiles are also comparable.

Comparability differs from homogeneity and, referring to academic and professional profiles, it is clear that diversity is not a draw back but an asset. The definition of professional profiles relates to the needs of society and social needs and demands are very varied. Hence consultation with social groups and the requests of professional bodies at either local, national or international level (in accordance to the aims of the degree) need to be taken into consideration. The Tuning project considers that consultations are important. It further recognises that these can be done in a variety of ways and in every case they should look for the most appropriate form and shape. This paper presents the findings of the consultations made by questionnaires because it was one used as a tool to initiate reflection on up-date information by the Tuning experts.

But the profiles are not only professional but also academic. Relating to academic institutions, degrees are expected to fulfil the requirements of the academic community be it at national and international levels. Looking for a common language to express academic and professional profiles, the Tuning project considers that the language of competences can be a useful common language for expressing comparability in terms of what the degree holders would be able to perform. It can also express common points of reference for the different subject areas, offering a non prescriptive framework of reference for the academic community (in this case the European Academic Community) and is a language which can be understood by European social groups, professional bodies and any other stake holders.

The Tuning Project is closely linked with the creation of the European Higher Education Area, and very explicitly with the Bologna process and the Prague Communiqué.

In relation to a system of easily readable and comparable degrees aimed at facilitating academic and professional recognition so that citizens can use their qualifications through the European HE Area, the introduction in Tuning sought to provide comparability and readability in reference to the competences (generic or subject-related) that the graduates from a particular degree aimed at attaining. In fact, the capacity to define which competences a programme seeks to develop, or what its graduates should be able to know, understand and do, cannot but add a further dimension to the degree transparency. They can also contribute to the development of both better-defined degrees, and systems of recognition that are "simple, efficient and fair", "reflecting the underlying diversity of qualifications" since competences add angles and levels, selecting knowledge appropriate to a particular profile. This works in favour of diversity.

As regards the adoption of a system essentially based on two main cycles:

The identification and initial discussion by a European body of academics of a set of subjectrelated competences for first and second cycle could be considered one of the major contributions of the project. In connection with knowledge, this is crucial for the development of European points of reference which could be considered common, diverse and dynamic in relation to specific degrees and the creation of frameworks of reference for clarification and further understanding of the relationship and nature of the qualifications.

Following on from this, joint reflection and work on competences and skills is an extremely important element in the work towards common standards and profiles for recognized joint 
degrees. Furthermore, the joint debate on the nucleus of competences and the articulation of levels and programmes by European networks can clearly enrich the European dimension of HE. It also builds on the consistency of systems of accreditation by increasing information on learning outcomes, and contributes to the development of common frameworks of qualifications, hence promoting understanding, clarity and the attractiveness of the European Higher Education Area.

An increase in transparency of learning outcomes and processes will definitely be a further asset for the encouragement and enhancement of mobility. Information which takes into consideration objectives expressed in the language of competences will present a more holistic perspective on the programme, but hopefully also will develop a systematic approach to each of the units in terms of the capacities which they will hope to gain. However, the specific contribution that Tuning seeks to offer relates particularly to the mobility of professionals and degree holders all over Europe, and has often been referred to as vertical mobility: the movement of graduates to take the second cycle of their studies in another country. In this respect the contribution of Tuning to the Diploma supplement is of great relevance.

Finally, along the different elements that made the European context where Tuning emerges is the adoption of common cornerstone of qualifications supported by a credit system such as ECTS, as accumulation and transfer

At the level of Programmes, elements which would be relevant to take into consideration: the European dimension of Higher Education in the different aspects mentioned above. They should also be able not only to use the European Credit System but to seek the links between credits and quality assurance:

- To link credits to quality assurance mechanisms in order to give them real application and thus 'currency' in the European area.

- Credits have a significant link to academic standards. In particular, the explicit identification of assessment criteria in relation to learning outcomes and teaching/learning methods is essential for any credit system. The examination of the relationship and articulation between these elements is highly significant for the maintenance of quality.

- The explanation of credits (in terms of curricular context: levels, learning outcomes, notional time and assessment regime) aids the precise explanation and vindication of standards. Without such definitions and links, credits remain simply crude statements about the volume of learning.

- International confidence in the quality of credits can only improve when national quality assurance mechanisms are rigorous, open, transparent and effective.

Focusing only on three aspects of quality: transparency, fitness-adequancy and relevance programme design in Higher Education could benefit from the approach of the Tuning project. However, these are only initial steps, initial remarks to open the debate.

\section{References}

ADAMS, S. (2003) Principles of a Pan-European Credit Accumulation Framework: Good Practice guidelines in Tuning Educational Structures in Europe - Final report. Gonzalez, J. and Wagenaar, R. Ed.) Deusto: University of Deusto and University of Groningen, pp. 220.

CAMPBELL, C. and VAN DER WENDE, M. (2000) International Initiatives and Trends in Quality Assurance for European Higher Education (Helsinki, The European Network for Quality Assurance in Higher Education).

EUROPEAN COMMISSION DG XXII. Comission Staff working paper, 2000. Council of the European Union, 2001. Confederation of European Union Rectors' Conferences, 2001. Thomas, E., 2000. Haug, G \& Touch, C., 2001. Mallea, J., 1998. Van Damme Dirk, 2001.

EUA European University Association (2001). Salamanca.

EUA European University Association (2003). Graz Reader. Strengthening the Role of Institutions. EUA Convention of European Higher Education Institutions. Graz, 29-31 May 2003 
GONZALEZ, J. and WAGENAAR, R. (Ed.) (2003). Tuning Educational Structures in Europe. Deusto: University of and University of Groningen.

GONZALEZ, J. and WAGENAAR, R. (2003) Tunning Educational Structures in Europe. Opportunities, Possibilities and Obstacles. Working on the European Dimension of Quality (Ministry of Education, Culture and Sciences, Zoetermeer).

GOSLING, D. and MOON, J. (2002) How to use Learning outcomes and assesment criteria (Seec, University of East London).

SURSOCK, A. (2003). Reflection from the Higher Education Institutions 'Point of View. Accreditation and quality culture. Working on the European Dimension of Quality (Ministry of Education, Culture and Science, Zoetermeer).

THOMAS, E. (2000). "Increading lifelong learning in European Higher Education: the challenges and the prospects". In F2000 European Higher Education Expert Forum, Brussels.

VAN DAMME, D. (1999) Internationalization and quality assurance: towards worldwide accreditation? Paper commissioned for the IAUP XII the Triannual Conference (Brussels).

VAN DAMME, D. (2001) European Quality Assurance: Developments and Challenges,

(Steering Committee of Tuning Project, Brussels).

VILLA, A. (2001) Marco Pedagógico de la Universidad de Deusto. Bilbao, University of Deusto.

WILLIAMS, P. (2002). QAA. Council of Universities. Transparency for European Higher Education. Madrid. 